\title{
Simulation of Ion Cyclotron Heating of Tokamak plasmas using coupled Maxwell and Quasilinear-Fokker-Planck solvers
}

\author{
M. Brambilla, R. Bilato \\ Max-Planck-Institut für Plasmaphysik-Euratom Association Boltzmannstrasse 2, \\ D-85748, Garching, Germany
}

\begin{abstract}
The code TORIC solving Maxwell equations in toroidal axisymmetric plasmas in the Ion Cyclotron (IC) frequency range has proven to be a useful tool for the simulation of IC heating and current drive in tokamak plasmas. TORIC has now been integrated in a package which includes, among other features, an interface to the experimental data (Grad-Shafranov MHD configuration, density and temperature profiles), interfaces to quasilinear Fokker-Planck solvers for the electrons and ions, and a subroutine which allows to estimate the effects of suprathermal anisotropic minority ions populations on wave propagation and absorption. This package allows somewhat simplified but essentially selfconsistent simulations of heating and current drive in this frequency domain. In this paper we summarize the physics included in TORIC, with particular emphasis on the most recent extensions, and present an example of application of the package to the analysis of two IC heating experiments in ASDEX Upgrade and in JET.
\end{abstract}




\section{The TORIC code.}

In this and the next section we briefly describe the code TORIC [1] and a few allied modules for the numerical simulation of plasma heating and current drive in the ion cyclotron (IC) range of frequencies. Our goal is to illustrate the physics included in the wave equations solved by TORIC, referring for the details as much as possible to already published documentation. These wave equations have been derived from the linearized Vlasov equation in toroidal geometry, by making clearly formulated approximations whose validity can be explicitly checked. Moreover, although the derivation is largely based on the assumption that the wavelengths of interest are large compared to the thermal ion Larmor radius (FLR approximation), enough large Larmor radius effects are included to allow the correct simulation of essentially all scenarios of interest.

\subsection{The model.}

TORIC solves the vector Helmholtz equation

$$
\vec{\nabla} \times \vec{\nabla} \times \vec{E}=\frac{\omega^{2}}{c^{2}}\left[\vec{E}+\frac{4 \pi i}{\omega}\left(\vec{J}_{\mathrm{P}}+\vec{J}_{\mathrm{A}}\right)\right]
$$

where $\vec{J}_{\mathrm{A}}$ is the antenna current, and $\vec{J}_{\mathrm{P}}$ is the high frequency (h.f.) plasma current. The ion contribution to $\vec{J}_{\mathrm{P}}$ is evaluated in the FLR approximation, following Swanson [2] and Colestock and Kashuba [3] (SCK approximation). The electron contribution is evaluated in the FLR approximation in the low-frequency limit $\omega \ll \Omega_{\text {ce }}$. The resulting wave equations describe damping by the ions at the fundamental ion cyclotron (IC) frequency and its first harmonic, and Cerenkov damping by the electrons (Landau and Transit Time damping, and the 'mixed' term discussed by Stix [4]). They also describe linear mode conversion of the externally excited fast wave (FW) near ion-ion resonances (and near $\omega=2 \Omega_{\mathrm{ci}}$ in a single species plasma) to short-wavelength hot-plasma waves, namely the lowest-order ion Bernstein wave (IBW) and/or the shear wave or its kinetic version, also known in this frequency range as quasi-electrostatic Ion Cyclotron wave (ICW) [5].

The h.f. plasma current of the SCK model is a second-order integro-differential functional of $\vec{E}$, first derived in plane-stratified geometry by [2] and [3] from the linearized Vlasov equation. Its expression for the tokamak has been obtained by writing this functional in vector form [6], and then transforming to axisymmetric toroidal coordinates [1]. In toroidal geometry, the wave equations are integral and non-local also in the FLR limit, because in the poloidal direction parallel dispersion also plays an essential role. Moreover, the evaluation of the orbit integrals along magnetic field lines in $\vec{J}_{\mathrm{P}}$ has to be modified to take into account toroidicity effects near IC resonances, as briefly discussed below. More recently [7], a direct derivation of $\overrightarrow{J_{\mathrm{P}}}$ in toroidal geometry has given the same result.

It is worth recalling that in the SCK model the ion h.f. current includes only the FLR contributions resonant at the first IC harmonic $\omega=2 \Omega_{\mathrm{ci}}$, while those resonant at 
the fundamental are omitted. The latter are always small corrections to the terms of order zero in the Larmor radius which are also resonant at $\omega=\Omega_{\mathrm{ci}}$. As a consequence, they have an appreciable influence on the local dispersion relation only in domains where the FLR expansion breaks down, namely around and below the IC resonance [8]. If the FLR corrections resonant at $\omega=\Omega_{\text {ci }}$ are kept in $\vec{J}_{\mathrm{P}}$, the lowest IB wave appears to remain propagative down to zero frequency, with a very short wavelength such that $k_{\perp} \rho_{i} \gg 1$, where $\rho_{i}$ is the thermal ion Larmor radius. This is contradicted by the full hot plasma dispersion relation, which predicts that no such wave exists below the lowest ion cyclotron frequency. The complete FLR expansion, moreover, falsifies the evaluation of fundamental IC damping, suggesting that power could locally flow from the ions to the waves even in a plasma in local thermal equilibrium. The SCK model does not suffer from either of these problems. On the contrary, the local dispersion relation of the SCK wave equations reproduces correctly the real part of the full hot-plasma index of the IC and IB branches, even in domains where the FLR approximation is formally not justified for these waves, and predicts the same fundamental IC damping as the full hot-plasma dispersion relation. We propose to call the SCK ion current, completed by the electron contribution as described above, the 'reduced' FLR expansion.

The resulting wave equations, however, miss electron Landau damping (ELD) of IB waves. To evaluate this damping, large ion Larmor radius effects must be taken into account. How this is done in TORIC is briefly recalled in subsection 1.3.

\subsection{Spectral representation of the fields.}

The solution of eqn (1) is assumed to be of the form (spectral representation)

$$
\vec{E}=\sum_{n_{\varphi}} e^{i n_{\varphi} \varphi} \sum_{m=-\infty}^{+\infty} \vec{E}^{m}\left(n_{\varphi} ; \psi\right) e^{i m \vartheta}
$$

where $\psi, \vartheta, \varphi$ are the radial, poloidal, and toroidal coordinates, respectively; for numerical convenience, $\psi$ is linear in the distance from the magnetic axis (hence $\psi \sim \sqrt{\Psi}_{P}$, the poloidal flux). TORIC takes advantage of axisymmetry to solve eqn (1) separately for each toroidal wavenumber $n_{\varphi}$ (a 'toroidal mode'). The superposition of different toroidal modes can be performed by a separate routine. The dependent variables in TORIC are the physical components of $\vec{E}$ with respect to a local magneticfield-aligned triad of unit vectors,

$$
\vec{u}_{\psi}=\vec{\nabla} \psi /|\vec{\nabla} \psi| \quad \vec{u}_{\eta}=\vec{u}_{\zeta} \times \vec{u}_{\psi} \quad \vec{u}_{\zeta}=\frac{\vec{B}_{0}}{B_{0}}
$$

Here $\vec{B}_{0}$ is the static magnetic field, and $\vec{u}_{\eta}$ points in the 'diamagnetic' direction. This choice considerably complicates the form of the differential operators in eqn (1), but is necessary because in the IC range $E_{\zeta}$ is smaller than $E_{\perp}$ by a factor $\sim m_{e} / m_{i}$ due to screening by the electrons. As a consequence, with any other choice of dependent variables $E_{\zeta}$ would be a small difference of two large quantities, and the evaluation of ELD would be affected by a large numerical noise. 
The form of the differential operators and of the various contributions to $\vec{J}_{\mathrm{P}}$ in the spectral representation are listed in [1]. The spectral ansatz transforms the integral operators into a generalized convolution. Thus, truncated at a sufficiently large poloidal number $m$, the representation (2) transforms Helmholtz equation into a (large) set of ordinary differential equations in the variable $\psi$ for the coefficients $E_{\alpha}^{m}(\psi)$ of the field (2). These equations are put into Galerkin weak-variational form, and discretized using cubic Hermite finite elements [9]. We might mention in passing that for the present problem this approach is free of spectral pollution [10] in the plasma, since in the FLR approximation eqn (1) has three independent solutions. This is not the case, however, in the vacuum layer between plasma and vessel; here the difficulty has been eliminated using an approach suggested by [11] (essentially, it amounts to ensure that $\vec{\nabla} \cdot \vec{E}=0$ is also satisfied). The spectral method, on the other hand, is only compatible with a simple idealized antenna lying in a surface of constant $\psi$ : it would be next to impossible, for example, to include in the simulation currents flowing in radial feeders and shorts. For this reason, the weights for the superposition of different toroidal modes are preferentially evaluated using the antenna code FELICE [12].

It is important to note that each poloidal Fourier mode in the spectral representation (2) of the field has a well-defined physical component of the wavevector in the 'parallel' direction,

$$
k_{\zeta}^{m n}(\psi, \vartheta)=\frac{m}{N_{\vartheta}} \sin \Theta+\frac{n_{\varphi}}{R} \cos \Theta \quad \tan \Theta=\frac{B_{\text {pol }}}{B_{\text {tor }}}
$$

where $N_{\vartheta}=g_{\vartheta \vartheta}^{1 / 2}$ is essentially the minor radius, and $R$ is the major radius. Since $k_{\zeta}^{m n}$ varies only on the equilibrium scale length, it plays locally the same role as $k_{\|}$in the uniform plasma limit in determining the local plasma response to the corresponding Fourier component of the field near collisionless particle-wave resonances. This circumstance allows to derive the wave equations used in TORIC from the linearized Vlasov equation in a rigorous way, essentially equivalent to the gyrokinetic approximation [7].

The dependence of $k_{\|}$on the wavenumbers $m$ and $n$, on the other hand, implies that each Fourier mode elicits a different response in the plasma. In this context, two important differences between the toroidal situation and a uniform or a planestratified plasma deserve to be stressed. In the first place, in the IC range of frequencies coupling between modes with different poloidal wavenumbers due to toroidicity is very strong, and can never be regarded as a perturbation. As a consequence, a quite large spectrum of poloidal modes is always simultaneously excited: in mode-conversion scenarios, several hundreds poloidal Fourier modes might be needed for the convergence of the representation (2), and even minority heating scenarios dominated by the longwavelength FW require several tens. In particular, therefore, it is not possible to characterize a toroidal mode by the single value $k_{\|} \sim n_{\varphi} / R$ : such an approximation is not even qualitatively adequate. This is illustrated in fig. 1, which shows the range of parallel wavenumbers and parallel phase velocities normalized to the electron thermal speed associated to the dominant toroidal mode $n_{\varphi}=12$ in a typical minority heating 

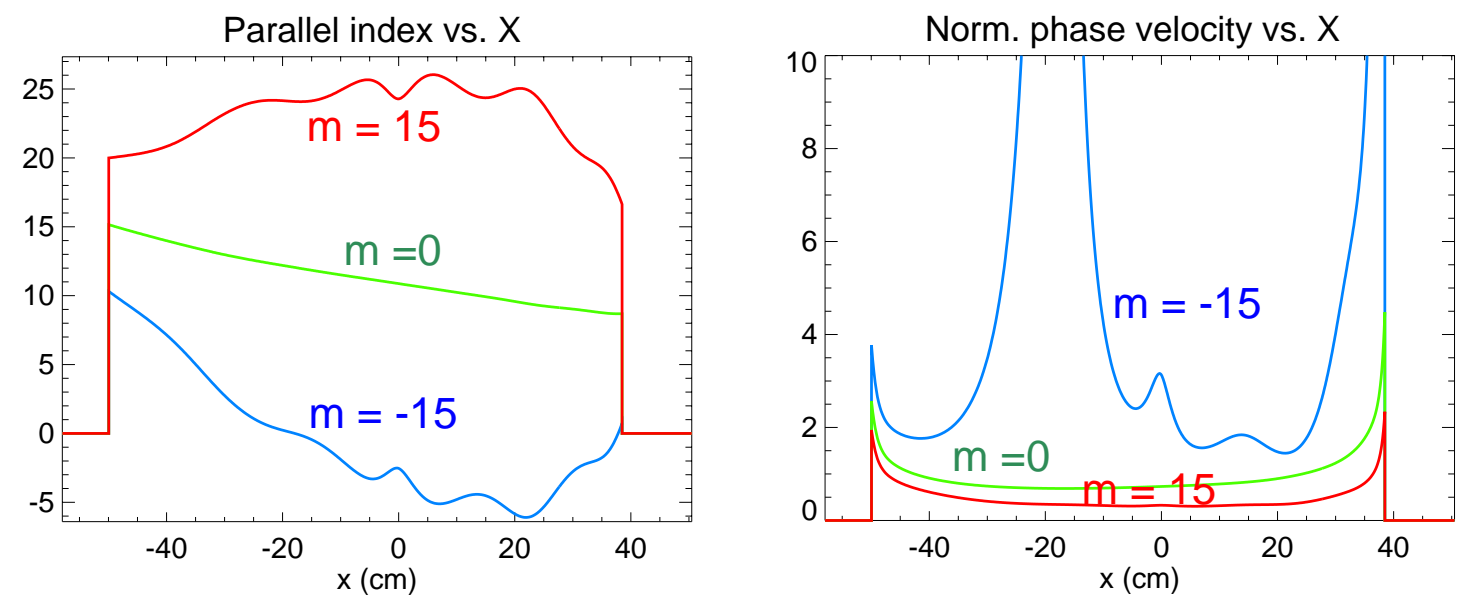

Figure 1. Parallel index and parallel phase velocity normalized to the electron thermal velocity along the equatorial plane, $n_{\varphi}=12$. The plasma parameters are those of Table 1.

scenario in ASDEX Upgrade [13]. The spread in mode conversion scenarios can be substantially larger.

The second toroidal effect taken into account in TORIC is the fact that near cyclotron resonances $\omega=p \Omega_{\mathrm{ci}}$ the width of the domain of non negligible parallel dispersion and absorption is influenced not only by the usual thermal Doppler effect, but also by the finite length of individual ion resonances [14]. This leads to a toroidicity induced broadening of cyclotron resonances, which is particularly important for modes with small $k_{\zeta}^{m n}$. This broadening, however, is evaluated assuming that all ions transit through resonance with nearly constant parallel velocity. This is not be entirely accurate, since quasilinear effects predict some accumulation of energetic trapped ions with turning point close to resonance.

\subsection{Large Larmor radius effects: Ion Bernstein waves.}

As mentioned above, a number of modifications to the FLR wave equations have been introduced in TORIC to take into account effects not adequately described by the reduced FLR model.

The FLR approximation is always well justified for the FW in the IC frequency range. The same is true also for the ICW, in spite of wavelengths of the order of the ion Larmor radius, because the plasma response to this wave is determined essentially only by the electrons. The situation is different, however, in the case of the IBW. This wave is excited by mode conversion at ion-ion resonances, and propagates towards the high-field side with rapidly decreasing wavelengths. As $k_{\perp} \rho_{i}$ approaches unity, refraction due to toroidicity causes a rapid upshift or downshift of $k_{\|}$, and thus (since IB waves are nearly electrostatic) a corresponding increase of $E_{\|}[15]$. When this occurs, efficient ELD of this wave is expected. While the reduced FLR expansion predicts with good accuracy the wavelengths of this wave also in domains where $k_{\perp} \rho_{i} \gg 1$, however, it misses ELD 
completely [8].

In principle, when $k_{\perp} \rho_{i} \gtrsim 1$, the IBW satisfies a complicated non-local integrodifferential wave equation. The failure of the FLR expansion to describe ELD of the IBW, however, occurs in a domain where the wavelength of this wave is so short that a WKB (Wentzell-Kramer-Brillouin) solution of the exact wave equation would certainly be adequate. It is possible to modify the FLR wave equations solved by TORIC in such a way that in the regions in question they admit for the IBW the same WKB solution, including damping. It is then legitimate to expect the solution evaluated by TORIC to be a good approximation to the solution of the exact problem. This has been confirmed by comparison with ray-tracing calculations [15]. The procedure involves adding to $\vec{J}_{\mathrm{P}}$ an appropriate differential contribution from the electrons, whose coefficients are evaluated by solving the full hot-plasma local dispersion relation (LDR) at each point and for each poloidal mode; the details can be found in [16]. The search for the IBW root of the LDR has been optimized so that this approach makes it possible to take into account large Larmor radius effects without altering the structure of the equations to be solved, and allows a fair description of propagation and absorption of IB waves in tokamak geometry with a relative modest numerical effort.

\subsection{Large Larmor radius effects: higher cyclotron harmonics.}

Large ion Larmor radius effects must be taken into account also in order to simulate IC damping of the FW at higher harmonics. In the high $\beta$ plasmas of NSTX [17], even the FW at a moderate IC harmonic has wavelengths comparable with the thermal ion Larmor radius. The strength of IC damping at $\omega=p \Omega_{\mathrm{ci}}$, moreover, is proportional to the modified Bessel function $I_{p-1}\left(k_{\perp}^{2} \rho_{i}^{2} / 2\right)$, and is, therefore, missed by the FLR expansion for $p \geq 3$.

To allow the simulation of FW IC damping at higher harmonics by TORIC, we have followed an approach similar to that implemented for the description of IB waves. Namely, when this option is chosen, the coefficients of the wave equations are modified in such a way that the differential equation solved by TORIC and the complete integral equation following from Vlasov equation admit the same local dispersion relation to all orders in the ion Larmor radius, this time for the FW. The details, and some application, can be found in [20]. This approach can be expected to give accurate results as long as it can be proven that the conditions for a local WKB approximation are satisfied by the FW: this is usually true, although not by such a large margin as in the case of IBWs. It is clear, however, that this assumption breaks down in the presence of a mode-conversion layer. As a consequence, scenarios in which the first $\left(2 \Omega_{\mathrm{ci}}\right)$ and higher IC harmonics are simultaneously present in the plasma cannot be simulated with TORIC. 


\section{The Fokker-Planck Quasilinear solver SSFPQL.}

In this section we briefly present the satellite routines which use the output of TORIC, in particular those which allow to estimate the production of suprathermal ion populations by IC heating, and their effects on wave propagation and absorption.

\subsection{Superposition of toroidal modes.}

As mentioned above, TORIC takes advantage of axisymmetry to solve the wave equations separately for individual toroidal modes $n_{\varphi}$. The fields and power deposition profiles of different toroidal modes add linearly, with weights depending on the antenna configuration. The subroutine SUMNPH performs such a superposition for the power deposition profiles, with weights obtained either from a simple antenna model, or from the output of the antenna code FELICE [12].

\subsection{Quasilinear diffusion coefficient for the electrons.}

The module QLDCE [21] uses the fields evaluated by TORIC to evaluate the bounceaveraged quasilinear diffusion coefficient $D_{\mathrm{QL}}^{e}$ for the electrons on each magnetic surface. Although in the FLR approximation the definition of $D_{\mathrm{QL}}^{e}$ is in principle entirely consistent with the evaluation of electron damping in TORIC, achieving consistency in the numerical implementation is far from trivial. In the IC range of frequencies the discreteness of the $k_{\|}$spectrum in tokamaks, combined with the toroidal localization of Cerenkov resonances, causes $D_{\mathrm{QL}}^{e}$ to be a very spiky function of $v_{\|}$. In turn, inaccuracies in the evaluation of $D_{\mathrm{QL}}^{e}$ are greatly amplified by the exponential variation of the number of resonant electrons as a function of $v_{\|}$. A special interpolation algorithm had to be developed to ensure that the quasilinear electrons heating rate evaluated with $D_{\mathrm{QL}}^{e}$ agrees within a few percent to the power deposited in the electrons according to TORIC.

The module QLDCE has been coupled to a Fokker-Planck solver for the electron distribution function developed in collaboration with Dr. I. Pavlenko, and used to estimate the efficiency of h.f. current drive by IC waves.

\subsection{A simple quasilinear Fokker-Planck solver for the ions.}

The module SSFPQL [22] solves the quasilinear equations for ions heated at the fundamental and the first cyclotron harmonic, using the output of TORIC to build the surface averaged quasilinear diffusion coefficient, and solves the resulting quasilinear kinetic equation on each magnetic surface. This code provides information on the radial profiles of the suprathermal populations generated by IC heating (distribution functions, perpendicular and parallel energies, number of fast ions), and on the collisional exchanges between these tails and the background ions and electrons. It should be noted, however, that SSFPQL is based on a rather simplified model. The main simplifications are: 
- The uniform-plasma Kennel-Engelmann quasilinear operator [23] is surfaceaveraged, neglecting several effects of toroidicity on IC heating, such as toroidal trapping, finite banana width, and losses.

- The collisional operator is linearized, assuming that the distribution of fast ions reaches steady state by losing energy on the background ions and electrons.

Exploiting the second assumption, SSFPQL solves directly for the steady-state, and is, therefore, very fast: the distribution functions of two ion species (minority heated at the fundamental, majority at the first harmonic) can be evaluated in less than $20 \mathrm{sec}$ on 100 magnetic surfaces on a laptop. Because of the simplified quasilinear operator, however, SSFPQL cannot be regarded as a full substitute for a more sophisticated Fokker-Planck solver or for Montecarlo simulations [24], particularly for the most energetic ions. For the bulk of the hot ion populations it nevertheless provides reliable information with a very modest computational effort.

\subsection{Iterating TORIC.}

In the basic option, TORIC evaluates the coefficients of the wave equations assuming Maxwellian distribution functions. Once the quasilinear distribution function of the minority ions has been evaluated by SSFPQL, however, the module QLMINH allows to iterate TORIC to estimate the influence of the suprathermal population on wave propagation and absorption.

The evaluation of the coefficients of the wave equations for arbitrary distribution functions involves multiple integrations in velocity space with resonant denominators, and can be orders of magnitude slower than the solution of the wave equations themselves. QLMINH short-circuits this problem by taking advantage of the fact that the distribution function of minority ions heated at the fundamental IC resonance can be approximated with reasonable accuracy as the superposition of two anisotropic Maxwellians, generalizing the well-known analytical approximations obtained by Stix [4]

$$
F_{\mathrm{m}}\left(v_{\|}, v_{\perp}\right)=(1-f) \frac{e^{-\left[\left(v_{\|}^{2} / \alpha_{\| 1}^{2}\right)+\left(v_{\perp}^{2} / \alpha_{\perp 1}^{2}\right)\right]}}{\pi^{3 / 2} \alpha_{\perp 1}^{2} \alpha_{\| 1}}+f \frac{e^{-\left[\left(v_{\|}^{2} / \alpha_{\| 2}^{2}\right)+\left(v_{\perp}^{2} / \alpha_{\perp 2}^{2}\right)\right]}}{\pi^{3 / 2} \alpha_{\perp 2}^{2} \alpha_{\| 2}}
$$

Here $f$ is the fraction of minority ions in the tail, and $\alpha_{\perp k}=\left(2 T_{\perp k}^{\mathrm{eff}} / m_{\mathrm{m}}\right)^{1 / 2}, \alpha_{\| k}=$ $\left(2 T_{\| k}^{\mathrm{eff}} / m_{\mathrm{m}}\right)^{1 / 2}$, with $T_{\perp k}^{\mathrm{eff}}$ and $T_{\| k}^{\mathrm{eff}}$ the effective perpendicular and parallel temperatures. With this approximate distribution function, the coefficients of the wave equations can be expressed in terms of the Plasma Dispersion Function $Z$, modified for toroidal effects as described in section 1 . The contribution of the minority species to the coefficients of $\vec{J}_{\mathrm{P}}$ to order 0 and 2 in the Larmor radius [1] are

$$
\begin{aligned}
& \delta \hat{L}=-\frac{\omega_{\mathrm{pm}}^{2}}{\omega^{2}} \sum_{k} f_{k} I_{0}\left(\lambda_{k}\right) e^{-\lambda_{k}}\left[-\gamma_{k} x_{0} Z\left(\gamma_{k} x_{1}\right)\right] \\
& \delta \hat{\lambda}_{i}^{(2)}=\frac{1}{2} \frac{\omega_{\mathrm{pm}}^{2}}{\Omega_{\mathrm{cm}}^{2}} \sum_{k} f_{k} \frac{\alpha_{\perp k}^{2}}{c^{2}} \frac{2 I_{1}\left(\lambda_{k}\right) e^{-\lambda_{k}}}{\lambda_{k}}\left[-\gamma_{k} x_{0} Z\left(\gamma_{k} x_{2}\right)\right] \\
& f_{1}=1-f, \quad f_{2}=f
\end{aligned}
$$


Table 1. Main parameters used in the simulations. The experimental profiles for $n$ and $T$ have been used for ASDEX Upgrade; the profiles of JET have been approximated analytically.

\begin{tabular}{llc}
\hline & AUG \# 19314 & JET \# 52095 \\
\hline Major radius & $1.67 \mathrm{~m}$ & $2.95 \mathrm{~m}$ \\
Plasma radius & $0.47 \mathrm{~m}$ & $0.85 \mathrm{~m}$ \\
Plasma composition & $6 \% \mathrm{H} \mathrm{in} \mathrm{D}$ & $4 \% \mathrm{H}$ in D \\
Central magnetic field & $1.97 \mathrm{~T}$ & $2.77 \mathrm{~T}$ \\
Ohmic current & $836 \mathrm{kA}$ & $2645 \mathrm{kA}$ \\
Central density & $6.5310^{19} \mathrm{~m}^{-3}$ & $3.810^{19} \mathrm{~m}^{-3}$ \\
Central electron temperature & $4.35 \mathrm{keV}$ & $8 \mathrm{keV}$ \\
Central ion temperature & $4.33 \mathrm{keV}$ & $8.0 \mathrm{keV}$ \\
Applied frequency & $30.5 \mathrm{Mhz}$ & $42.0 \mathrm{Mhz}$ \\
Representative toroidal wavenumber & $n_{\varphi}=12$ & $n_{\varphi}=24$ \\
Position of minority resonance (r/a) & $0.197($ h.f.s. $)$ & $0.070($ h.f.s. $)$ \\
Estimated total power coupled & $4 \mathrm{MW}$ & $9 \mathrm{MW}$ \\
Peak power density & $2.5 \mathrm{~W} / \mathrm{cm}^{3}$ & $2.5 \mathrm{~W} / \mathrm{cm}^{3}$ \\
\hline
\end{tabular}

where $x_{p}=\left(\omega-p \Omega_{\mathrm{c} \alpha}\right) / k_{\|} v_{\mathrm{th} \alpha}, \quad \gamma_{k}=v_{\text {th } \alpha} / \alpha_{\| k}, \lambda_{k}=k_{\perp}^{2} \alpha_{\perp k}^{2} / 2 \Omega_{\alpha}^{2}$ with $k_{\perp}$ the perpendicular wavenumber of the $\mathrm{FW}$, and the other notations are standard. The factors involving the modified Bessel functions take into account large Larmor radius effects in the presence of energetic suprathermal tails. Iterating TORIC including these contributions only involves a larger number of evaluations of the function $Z$ and of Bessel functions, for which purpose efficient algorithms are available.

The parameters of the representation (5) are determined by matching as well as possible the logarithmic slopes in the parallel and perpendicular velocity, and the energy content of the quasilinear distribution evaluated by SSFPQL. Accurate matching of the slopes is particularly important, since IC absorption is proportional to velocity derivatives of the distribution function. From eqns (6) it is also clear that the effective parallel and perpendicular temperatures play a very different role in the wave equations. Only the former influences the Doppler width of the resonance. An increasing effective perpendicular temperature decreases slightly the strength of fundamental IC absorption, and increases the wavelength of IB waves excited by mode conversion. It is, therefore, important to know the anisotropy of the minority distribution function, which is a sensitive function of the local power density and collisionality. Notwhistanding its limitations, SSFPQL is perfectly adequate to provide this information.

\section{Hydrogen minority heating in ASDEX Upgrade.}

As an example, we have used TORIC to simulate minority heating of Hydrogen in a Deuterium plasma in the shot 19314 of ASDEX Upgrade (AUG). The parameters of the experiment are summarized in Table 1. The experimentally determined MHD equilibrium and the density and temperature profiles measured at $t=3.2 \mathrm{sec}$ have been 

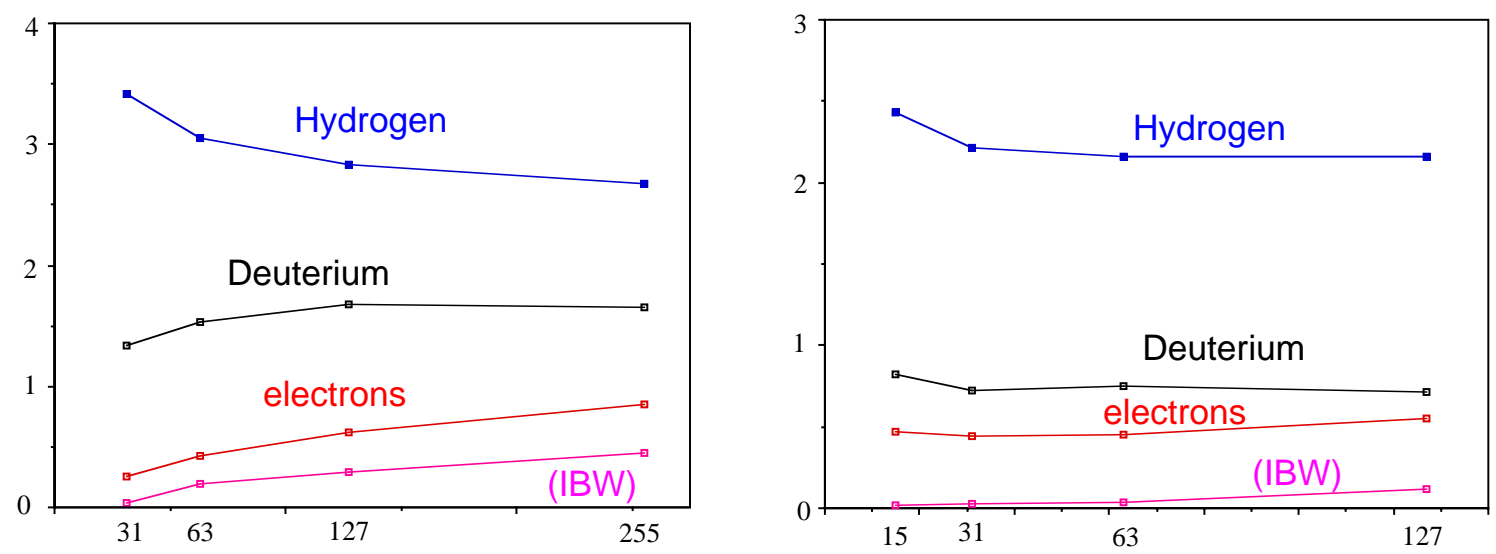

Figure 2. Convergence investigation with the number of poloidal modes: left $n_{\varphi}=1$, right $n_{\varphi}=6$. The ordinate is the total absorption in each channel (im MW) per $1 \mathrm{kA}$ of current in the corresponding $n_{\varphi}$ mode of the antenna.

used. The estimated $\mathrm{H}$ concentration has been assumed radially uniform, and higher $\mathrm{Z}$ impurities have been neglected.

\subsection{Convergence investigation.}

As a preliminary, it is instructive to discuss briefly the results of a study of the convergence of the field evaluation with respect to the number $M$ of poloidal Fourier modes (in all the runs $M=2^{N-1}-1$, where $2^{N}$ is the number of points in the poloidal mesh, so that the poloidal wavenumber $m$ scans the range $-(M-1) / 2 \leq$ $m \leq(M-1) / 2)$. Since the Doppler width of IC resonances is roughly proportional to the toroidal wavenumber $n_{\phi}$, modes with small $n_{\phi}$ are expected to require a finer poloidal mesh, and correspondingly a larger $M$. This is confirmed by the variation of the absorption in the different channels vs M, presented in fig. 2.

The predicted fundamental IC absorption by $\mathrm{H}$ decreases somewhat with increasing $M$, while harmonic IC absorption by $\mathrm{D}$ increases, and electron damping, although always small, increases even faster. This behaviour is easily related to the physics of the different damping mechanisms. Harmonic IC and electron damping are weighted towards large poloidal numbers, because the former is a Finite Larmor radius effect, and the latter requires slow parallel phase velocities (high $m$ ). Damping of IB waves, moreover, is at the same time a large Larmor radius effect. Since fundamental and harmonic IC damping require an adequate sampling of the absorption region, on the other hand, one would expect both these channels to increase with $M$, at least initially. There is, however, a compensating effect, namely that $\left|E_{+}\right|$must have a rather marked minimum at the minority resonance, which requires enough poloidal modes to interfere destructively at the right position. Insufficient screening of $E_{+}$at low poloidal resolution can explain why fundamental IC damping actually decreases with increasing $M$. Harmonic damping, being a FLR effect, depends on the derivatives of $E_{+}$, and is, therefore, much less sensitive to the accuracy of the interference in question, and 


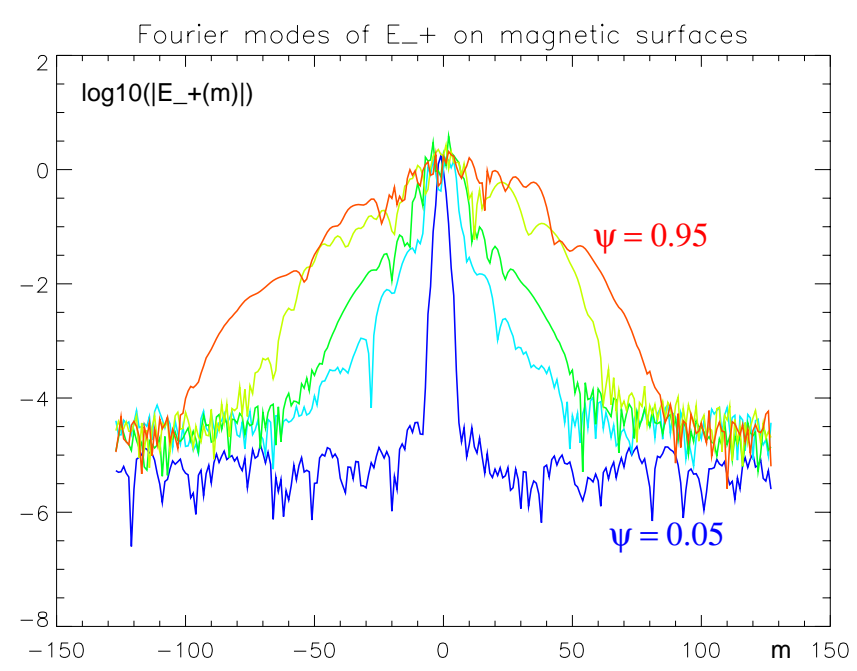

Figure 3. Poloidal Fourier spectrum of $\left|E_{+}(m)\right|$ on 5 equidistant magnetic surfaces, ASDEX Upgrade plasma, $n_{\varphi}=1$. The width of the spectrum increases monotonically from the axis to the edge.

more to the inclusion of sufficiently large values of $m$.

At $n_{\varphi}=1,31$ poloidal modes seem to be about sufficient to produce the required minimum of $\left|E_{+}\right|$(in fact, there is no significant visual difference in the field plots between the 4 runs of this scan). Because of the other requirements, the run with 127 modes is acceptably, and the run with 255 modes well converged. The latter statement is confirmed by fig. 3, which shows a logarithmic plot of the amplitudes of the Fourier modes of $E_{+}$for $n_{\varphi}=1$, on 5 equidistant magnetic surfaces, $0.05 \leq r / a \leq 0.95$. This figure also illustrates the fact that problems of numerical resolution are always more severe near the plasma edge, as it is to be expected for geometrical reasons. With $n_{\phi}=6$, convergence is acceptable with $M=63$, and good with $M=127$. As a consequence of this convergence study, in the scan over toroidal modes presented next, we have used 255 poloidal modes for $n_{\varphi}=1,127$ for $2 \leq n_{\varphi} \leq 6,63$ for $7 \leq n_{\varphi} \leq 12$, and 31 for $\left|n_{\varphi}\right| \geq 13$.

It is worth mentioning here that the relatively low number of poloidal modes required for an adequate representation of $\vec{E}$ in the resonance region is typical of Hydrogen minority in Deuterium. In this scenario, because of the simultaneous resonance of the majority FLR contributions to the hf current at $\omega=2 \Omega_{c D}$, the poloidal variation of $E_{+}$in the layer between IC resonance and mode conversion is relatively smooth. In other scenarios, such as Helium ${ }^{3}$ minority, the variation is much sharper, and many more modes may be needed. A convergence check as sketched here is then particularly important, since using incompletely converged runs of TORIC in these scenarios can lead to grossly overestimate the heating of the minority. As an example, we show in fig. 4 the fraction of power predicted to be absorbed by the $\mathrm{He}^{3}$ minority in a deuterium plasma in ASDEX Upgrade, as a function of concentration, for $n_{\phi}=6$ and 12 (current drive and and heating antenna configurations, respectively). To obtain the correct transition from minority to mode conversion regimes when $n_{H e^{3}} / n_{e}$ exceeds 


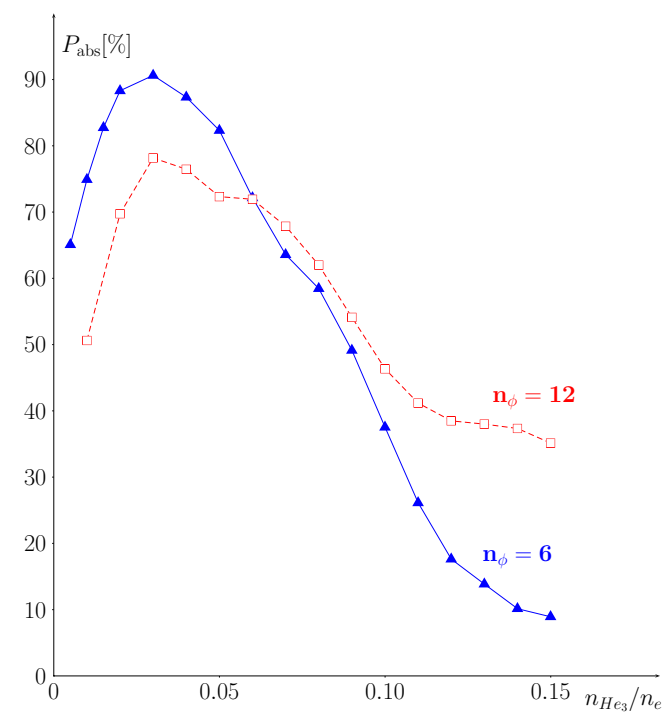

Figure 4. Fraction of power absorbed by $\mathrm{He}^{3}$ minority in Deuterium, versus concentration, in ASDEX Upgrade. Central magnetic field $2.7 \mathrm{~T}, \mathrm{f}=30.5 \mathrm{Mhz}$, $n_{e}(0)=510^{19} \mathrm{~m}^{-3},, T_{e}(0)=T_{i}(0)=4 \mathrm{keV}$.

$\sim 4 \%$, in agreement with the theoretical [18] and experimental [19] expectations, it was necessary to run the parallelized version of toric with a mesh of 512 poloidal points and 255 poloidal Fourier modes. With the finest mesh practical on a serial computer (128 points and 63 modes) the field pattern appears already to some extent acceptable, but the predicted absorption by the $\mathrm{He}^{3}$ in the mode conversion domain remains roughly constant at high level as a result of incompete screening of $E_{+}$at $\omega=\Omega_{c e_{3}}$.

\subsection{Full scan over toroidal modes.}

Figure 5 shows the coupled power spectrum predicted by the FELICE code [12], and the repartition of power among the different absorption channels, evaluated by TORIC and weighted with the spectrum produced by FELICE, for the ASDEX Upgrade case of Table 1. The two strap antenna is excited in the antisymmetric configuration; for simplicity, we have neglected the slight asymmetry between positive and negative values of $n_{\varphi}$ which nevertheless exists due to toroidicity. In running FELICE, outward radiation conditions have been imposed before the IC resonance layer, explaining the smoothness of the spectrum obtained. Because of the plane-stratified geometry approximation, when the integration is extended over the entire plasma column until the "opposite" metallic wall FELICE tends to overestimate the quality of internal eigenmodes. As a consequence, imposing outward radiation conditions often predicts a loading resistance 


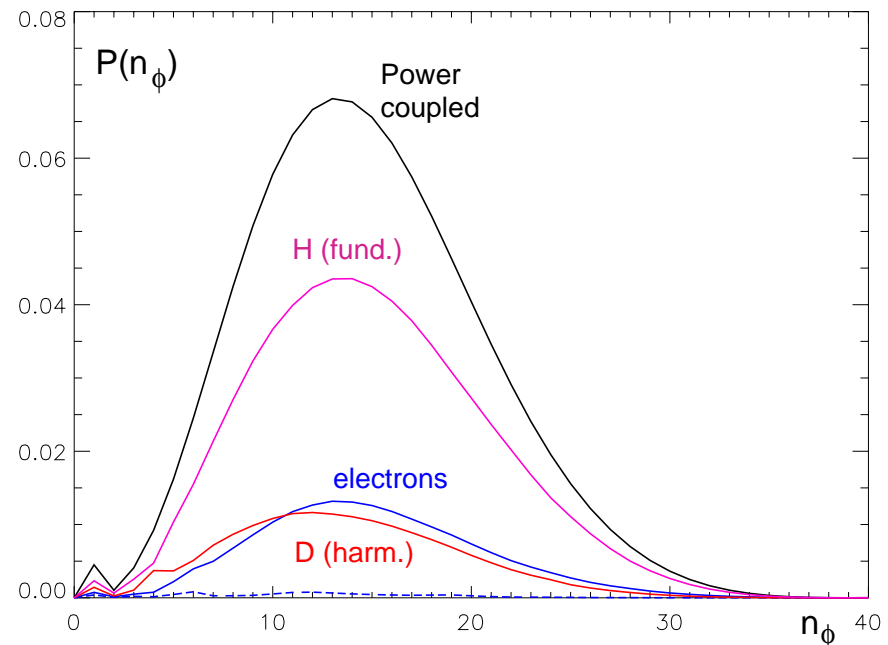

Figure 5. Toroidal power spectrum. The total coupled power is normalized to unity. The coupled spectrum is evaluated by FELICE, the fraction absorbed in each channel by TORIC.

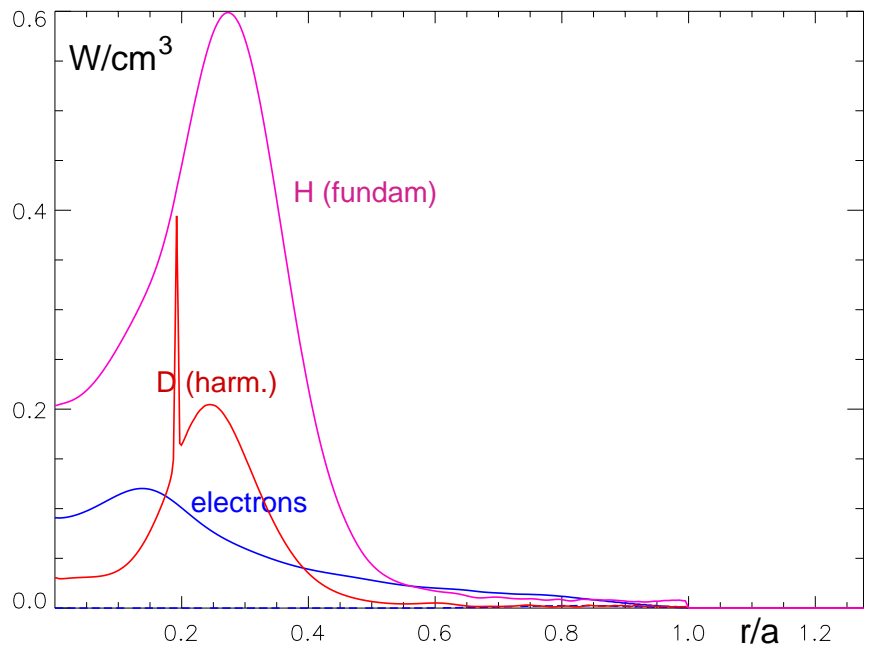

Figure 6. Radial power deposition profiles evaluated by TORIC, and weighted with the coupled spectrum of FELICE, including the toroidal modes $0 \leq n_{\varphi} \leq 40$. The total coupled power is normalized to $1 \mathrm{MW}$. The spike in harmonic damping of D occurs where the resonance cuts the equatorial plane, and might be spurious.

in better agreement with the experimental observations. In this case, however, the absorption per transit is sufficiently strong to largely suppress internal modes excitation even in the limit of straight geometry. The spectrum obtained by integrating over the entire plasma, therefore, although presenting some more structure, does not differ significantly from the one shown in fig. 5 .

Figure 6 shows the corresponding power deposition profiles. These profiles, multiplied by the estimated power of 3.6 MW actually coupled in the experiment, are used by SSFPQL to evaluate the quasilinear ion velocity diffusion coefficient as function of radius. Figure 7 shows the minority (Hydrogen) distribution function at the point 


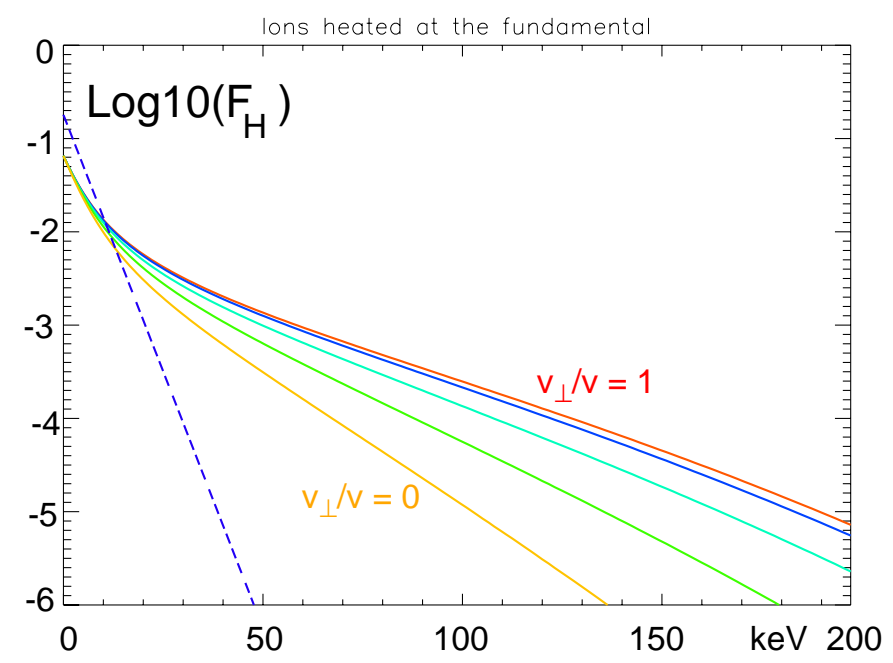

Figure 7. AUG experiment: Minority distribution function at $r / a=0.25$, for a total coupled power of 3.6 MW. The dashed curve is the unperturbed Maxwellian at this radius, with a temperature of $3.83 \mathrm{keV}$.
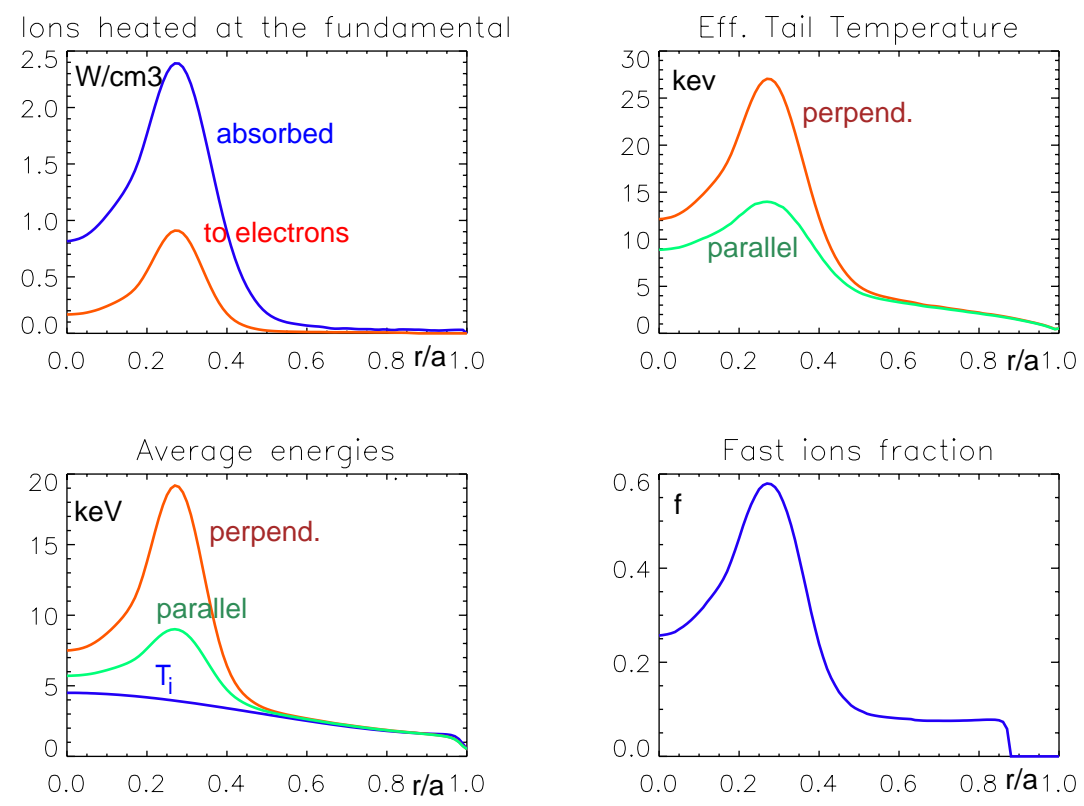

Figure 8. AUG experiment: power absorbed by $\mathrm{H}^{+}$and collisionally transferred to the electrons, and parameters of the minority distribution function vs radius, for a total coupled power of $3.6 \mathrm{MW}$.

where the absorbed power density is maximal, $r / a \sim 0.25$. The effective perpendicular temperature (logarithmic energy derivative at $v_{\|}=0$ ) of the tail is about $28 \mathrm{keV}$, in good agreement with the charge exchange measurement of $30-35 \mathrm{keV}$ [25]. The parallel effective temperature of the tail is about half the above value, $12.5 \mathrm{keV}$. Finally, fig. 8 shows the power collisionally transferred to the electrons, and the parameters of the minority distribution function evaluated by fitting the representation (5) to the results of SSFPQL. Because of the modest effective temperature of the suprathermal tail, most 

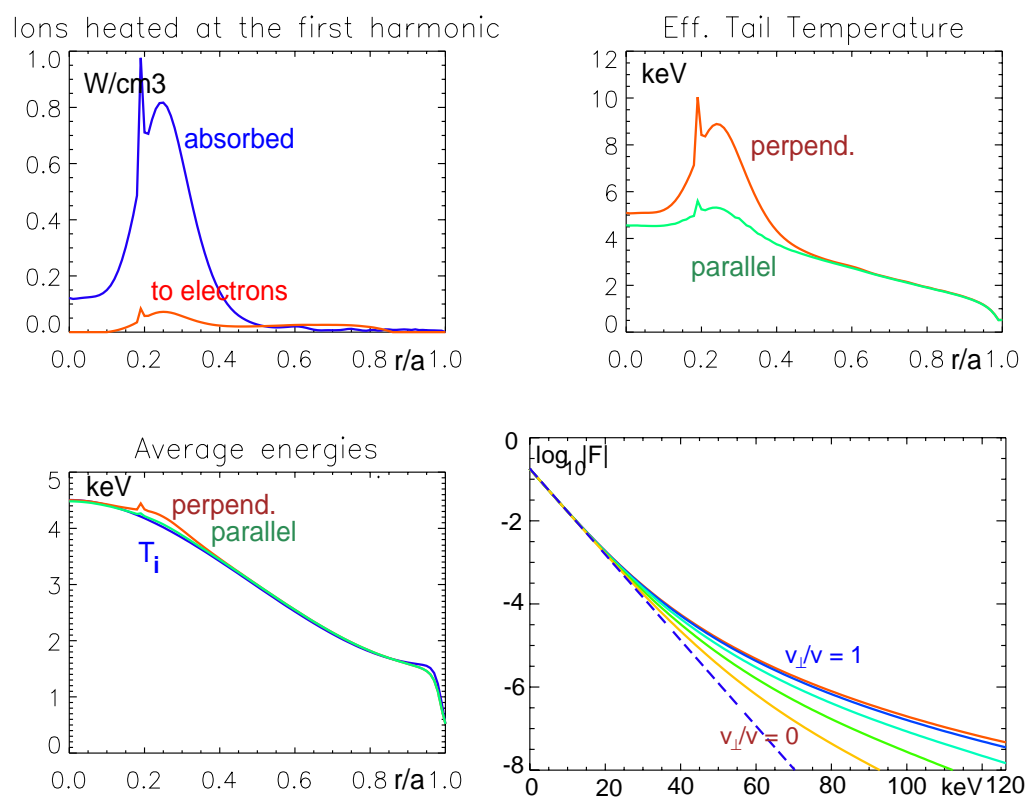

Figure 9. AUG experiment: power absorbed by $\mathrm{D}^{+}$and collisionally transferred to the electrons, parameters of the majority distribution function vs radius, and distribution function at $r / a=0.25$, for a total coupled power of 3.6 MW.

of the power absorbed by the protons from the waves is collisionally transferred to the background ions: integrated over the volume, less than $30 \%$ goes to the electrons, namely about $10 \%$ by direct absorption, and the rest by collisional transfer. In the experiment no substantial electron heating during the h.f. power pulse was observed [30].

The same results are shown in fig. 9 for the majority species. The power per ion directly absorbed by Deuterium is more than one order of magnitude less than for Hydrogen. Thus not only the effective tail temperatures are much lower, but the contribution of suprathermal deuterons to the total energy is minimal, indicating that their number is very small. This is confirmed by the details of the Deuterium distribution function: discrepancies from the unperturbed Maxwellian begin at a much higher energy than in the case of Hydrogen. This is also a consequence of the well-known fact that harmonic heating is a finite Larmor radius effect.

\subsection{Quasilinear effects on power absorption.}

In the present case, the effect of suprathermal ions on the power deposition profiles is quite modest. In fig. 10 we compare the profiles for the toroidal mode $n_{\varphi}=12$ (at the peak of the power spectrum) for the unperturbed Maxwellian plasma, and taking into account the quasilinear Hydrogen distribution function evaluated by SSFPQL using the approach outlined in section 2. Some increase and broadening of the ion absorption at the expense of electron heating is visible. Since the effect is small and limited to the central region, however, the changes in the global power balance integrated over the plasma volume are of the order of one per cent only. 

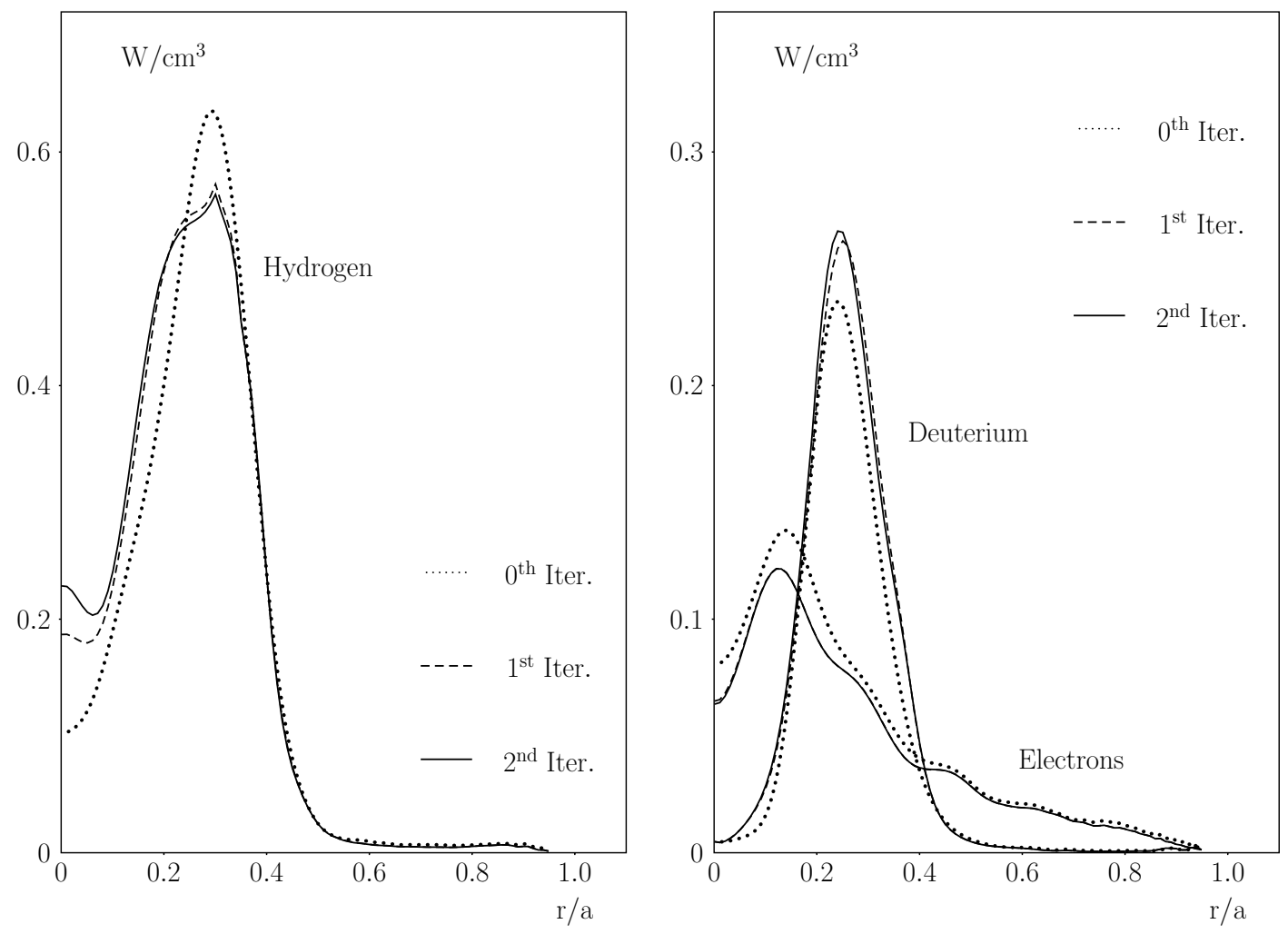

Figure 10. Power deposition profiles of the toroidal mode $n_{\varphi}=12$ in the unperturbed Maxwellian plasma (dotted curves) and with the quasilinear minority distribution function evaluated by SSFPQL (dashed and full curves) for the AUG experiment. The ordinates are $\mathrm{W} / \mathrm{cm}^{2}$ normalized to a total power of $1 \mathrm{MW}$, the power coupled 3.6 MW.

\subsection{Selecting a representative toroidal mode.}

It is common practice to run TORIC for a single value of $n_{\varphi}$, corresponding to the peak of launched power, and regard the results as representative for the entire spectrum. Clearly, this procedure is best justified for 'selective' antennas with 4 or more straps. Comparing the profiles of fig. 9 with those of fig. 5 shows, however, that in many situations it can be safely used even in the case of a simple two-strap antenna. The profiles weighted over the whole spectrum are slightly broader than those for the mode $n_{\varphi}=12$ alone, but neither the absolute values nor the shapes are substantially different. This can be understood from well-known properties of the FW. Namely, IC absorption, integrated over the Doppler width of the resonance, depends quite weakly on $k_{\|}$. This is not true, of course, for absorption by the electrons; due to the dependence of $k_{\|}$on the poloidal number $m$, however, even a single toroidal modes excites a sufficiently broad range of parallel phase velocities to be representative of the whole spectrum, at least as a first approximation (cfr. fig. 1). The largest discrepancy can be expected near the magnetic axis, where only the mode $m=0$ survives to contribute to ELD and TTMP; this is also confirmed by the comparison of the electron profiles in fig. 5 and 9 . 


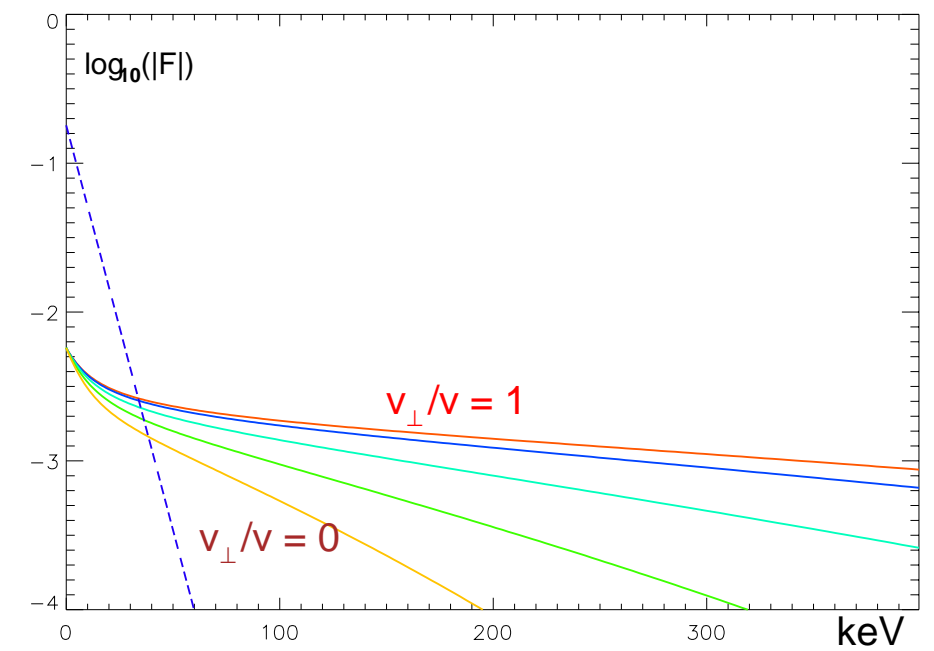

Figure 11. JET experiment: Minority distribution function at $r / a=0.25$, for a total coupled power of 3.6 MW. The dashed curve is the unperturbed Maxwellian at this radius, with a temperature of $8 \mathrm{keV}$.

\section{Hydrogen minority heating in JET.}

In contrast to the AUG results, in a similar experiment on the JET tokamak [26] strong electron heating was observed. The AUG and JET scenarios were quite similar: low minority concentration well within the minority regime, and almost identical coupled power densities (the main parameters of the JET experiment are summarized in the right column of Table 1). Simulations with TORIC and SSFPQL reproduce well the observations, and allow to ascribe the different outcome to the lower collisionality and somewhat lower Hydrogen concentration of the JET plasma.

Taking advantage of the remark at the end of the previous section, in the JET case only the most representative toroidal mode $n_{\varphi}=24$ has been taken into account. The results of TORIC and SSFPQL for the minority are shown in figs 11 and 12 . The absorbed power density in the central region is comparable to that in the AUG experiment: the much larger plasma volume in JET is compensated by the larger total power available, and by a better focusing of the waves in the central region. In JET, however, the predicted effective temperatures of the minority suprathermal population are much higher, and more anisotropic: $T_{\perp \text { eff }} \simeq 380 \mathrm{keV}, T_{\| \text {eff }} \simeq 60 \mathrm{keV}$ (fig. 12). Except for minor differences in the power deposition profiles, these results are consistent with the analysis made in [26]. The critical temperatures for preferential slowing down on electrons are about $90 \mathrm{keV}$ in ASDEX Upgrade, and $210 \mathrm{keV}$ in JET. As a consequence of the high effective tail temperatures, in the core of JET $\sim 80 \%$ of the power absorbed by the minority is thermalized on the electrons $(\sim 65 \%$ integrated over the entire plasma). The difference with the AUG case is due to the larger power per minority ion available in JET (both the total density and the minority concentrations being lower), and to the lower collisionality (by about a factor 4) of the JET plasma. Simulations scanning the total power and the minority concentration in the two devices confirm this 

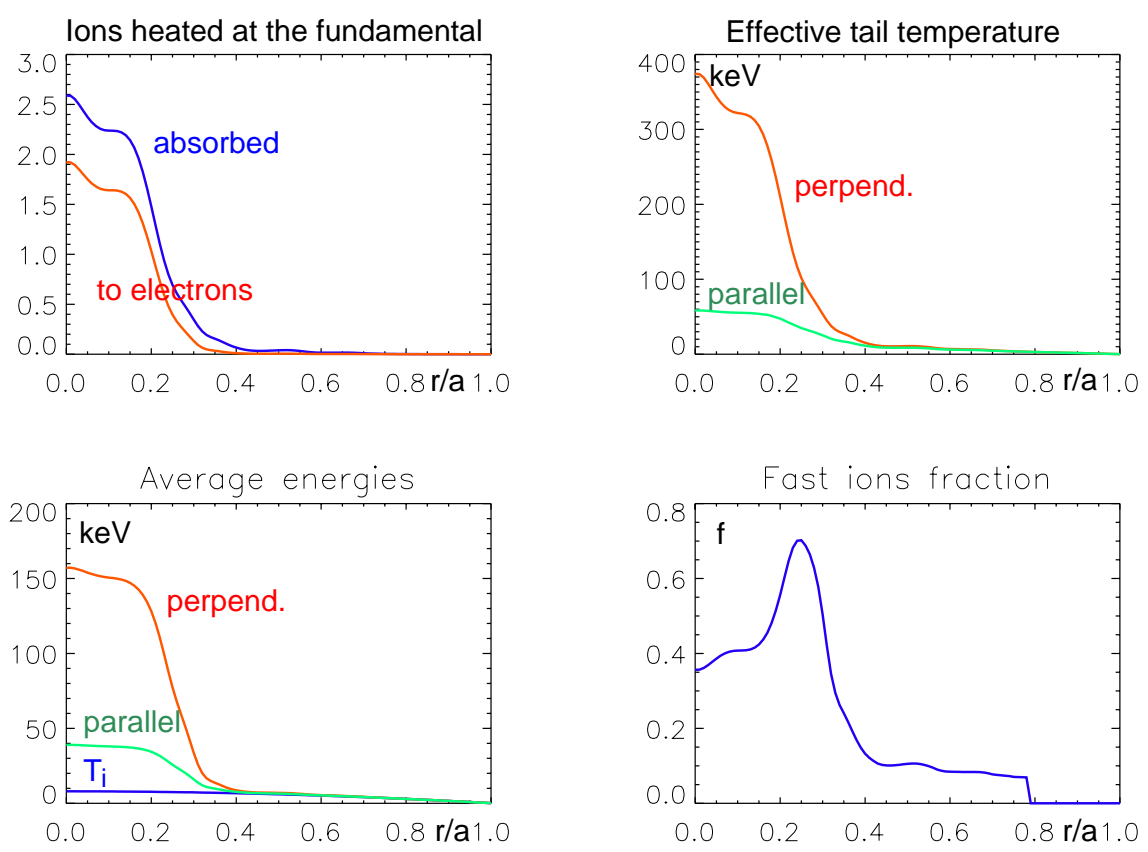

Figure 12. Jet experiment: power collisionally transferred to the electrons, and parameters of the minority distribution function vs radius, for a total coupled power of 9 MW.

interpretation.

The result of iterating TORIC for this JET scenario are shown in fig. 13. They are similar to those for ASDEX Upgrade, except that the differences between the Maxwellian and the quasilinear case are somewhat larger. It is interesting to note that in both cases the peak absorption by the minority decreases when the suprathermal tails are taken into account. In the JET example, the total h.f. power absorbed by the minority decreases from $\sim 72.3 \%$ in the Maxwellian plasma to $\sim 66.5 \%$ in the quasilinear case. This is in agreement with the fact that the Bessel function $I_{0}\left(\lambda_{k}\right)$ in eqn 6 is a decreasing function of the average perpendicular particle energy. Replacing $I_{0}\left(\lambda_{k}\right)$ by unity in the simulations, however, shows that only about half of the difference between the Maxwellian and the quasilinear case is due to large Larmor radius effects.

It should also be mentioned that the slight broadening of the minority absorption profile predicted by TORIC in the quasilinear case takes into account only the increased width of the resonance due to the larger $T_{\| \text {eff }}$. In JET, the perpendicular energy of the ions in the tails is so large that the thin banana orbit approximation required to justify surface averaging the quasilinear equation becomes questionable. Describing the effects of finite orbit width on the IC absorption profiles requires a more sophisticated approach [24]. 

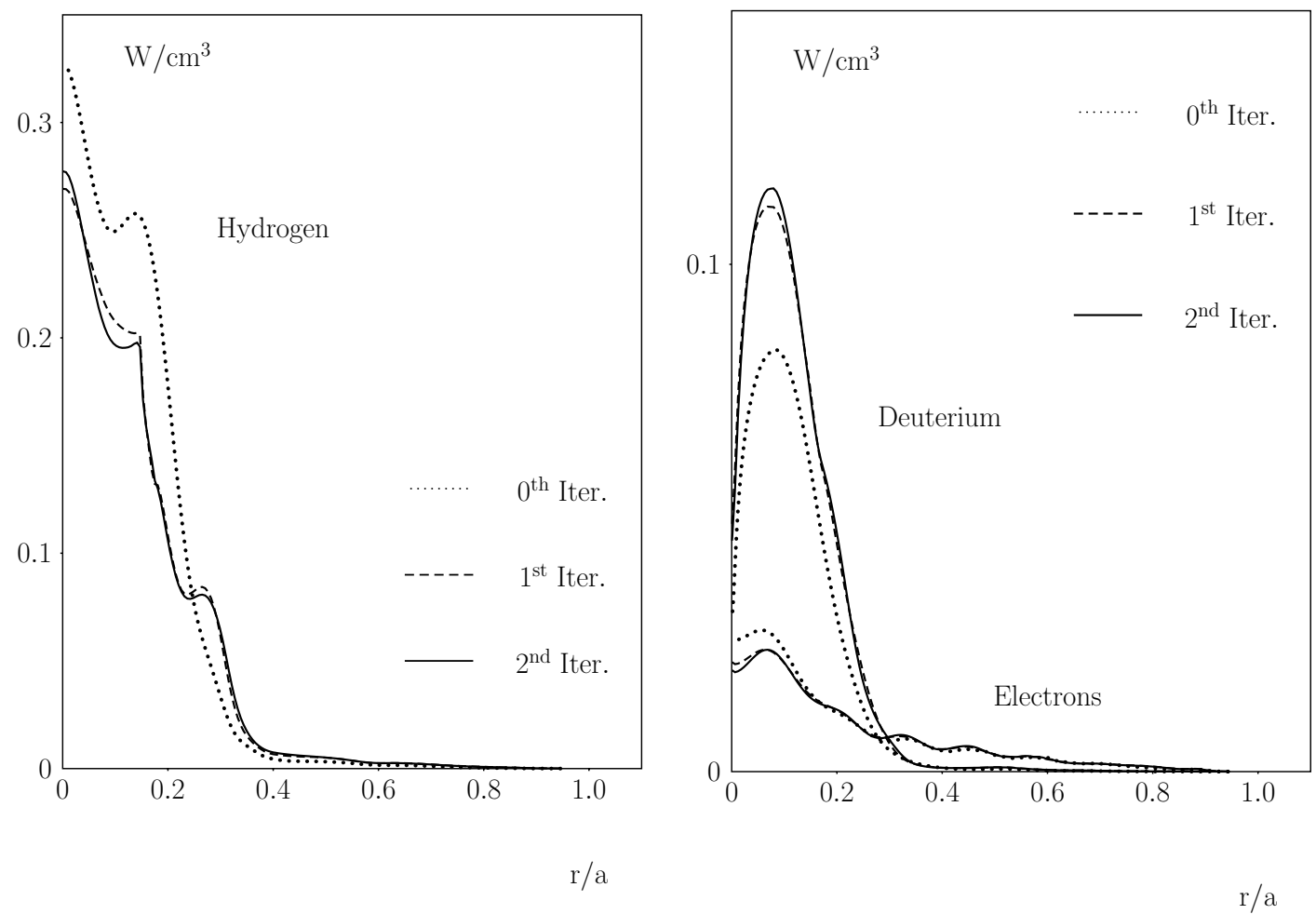

Figure 13. JET experiment: power deposition profiles of the toroidal mode $n_{\varphi}=24$ in the unperturbed Maxwellian plasma (dotted curves), and with the quasilinear minority distribution function evaluated by SSFPQL (dashed and full curves). The ordinates are $\mathrm{W} / \mathrm{cm}^{2}$ normalized to a total power of $1 \mathrm{MW}$, the power coupled was $9 \mathrm{MW}$.

\section{Comments and conclusions.}

We have presented the models on which the full-wave toroidal code TORIC and the quasilinear Fokker-Planck solver SSFPQL are based, and we have summarized the physics which can be investigated by combining the two codes. The wave equations solved by TORIC are based on the FLR approximation, but take into account all large Larmor radius effects as required for the correct simulation of essentially all scenarios of interest. SSFPQL neglects a number of interesting toroidal effects, most notably the accumulation of energetic trapped ions with turning point just inside resonance, which can be observed in more detailed quasilinear simulations. As mentioned in section 4, however, the main source of inaccuracy is the fact that SSFPQL, in common with most surface averaged Fokker-Planck solvers, neglects the finite radial width of the orbits of the most energetic ions. Nevertheless, simulations with the combined TORIC and SSFPQL codes reproduce satisfactorily the essential experimental observations, and help in their interpretation, with a modest numerical effort. The output of SSFPQL, as presented in figs 7,8 , and 10, can directly be used as input to transport codes. 


\section{Acknowledgments}

TORIC has benefitted of the contributions by several persons. A first version [27] was written in collaboration with Dr. T. Krücken. Dr. P. Bonoli has written a module for the estimation of the efficiency of h.f. current drive based on the Karney-Ehst parameterization [28], helped in the development of a more efficient solver for the stiffness matrix, and suggested several important improvements. The interface with numerical MHD equilibria has been made possible by the help of Dr. D. McCune and Dr. F. Meo. Dr. D. McCune has installed TORIC in the transport package TRANSP. Dr. E. D'Azevedo and Dr. J.C. Wright have parallelized the code, making it possible to simulate large plasmas and scenarios which require very fine mesh for convergence [29].

We also wish to thank H.-U. Fahrbach, C. Maggi, J.-M. Noterdaeme, W.S. Suttrop, and the ASDEX Upgrade Team for informations and discussions on the IC experiment in ASDEX Upgrade [30].

\section{References}

[1] M. Brambilla, Plasma Phys. Contr. Fusion 41, 1, (1999).

[2] D.G. Swanson, Phys. of Fluids 24, 2035, (1981).

[3] P.L. Colestock, R.J. Kashuba Nucl. Fusion, 23, 763, (1983).

[4] T.H. Stix, Nucl. Fusion 15, 737, (1975).

[5] F.W. Perkins, Nucl. Fusion 17, 1197, (1977).

[6] M. Brambilla, Plasma Phys. Contr. Fusion 31, 723, (1989).

[7] M. Brambilla, Plasma Phys. Contr. Fusion 41, 775, (1999).

[8] M. Brambilla, Kinetic Theory of Plasma Waves, Oxford Univ. Press, (1998).

[9] K. Appert, T. Hellsten, J. Vaclavik, and L. Villard, Comp. Phys. Comm. 40, 73, (1986).

[10] X. Llobet, K. Appert, A. Bondeson, J. Vaclavik, Comp. Phys. Comm. 59, 199 (1990).

[11] Bo-Nan Jang, Jie Wu, and L.A. Povinelli, J. Comp. Phys. 125, 104, (1996)

[12] M. Brambilla, Plasma Phys. Contr. Fusion 35, 41, (1993).

[13] J.-M. Noterdaeme et al., 26th EPS Conf. Contr. Fusion and Plasma Phys., Maastricht 1999, 23J, 1561, (1999).

[14] M. Brambilla, Phys. Letters A 188, 376, (1994).

[15] M. Brambilla, 27th EPS Conf. on Contr. Fusion and Plasma Physics, http://epsppd.epfl.ch/Buda/pdf/P4_105.pdf

[16] M. Brambilla, Nucl. Fusion 38, 1805, (1998).

[17] M. Ono et al., Nucl. Fusion 40, 557, (2000).

[18] S.C. Jun, Kaya Imre, D.C. Stevens, H. Weitzner, and C.S. Chang, Phys. of Plasmas 7, 1467 (2000).

[19] M. Porkolab, C. Fiore, M. Greenwald, J.C. Hosea, et al, 13th Topical Conf. on Radio Frequency Power in Plasmas, Annapolis 1999, (AIP Conf. Proc. 485, S. Bernabei and F. Paoletti eds.), p. 79

[20] M. Brambilla, Plasma Phys. Contr. Fusion 44, 2423, (2002).

[21] R. Bilato, M. Brambilla, I. Pavlenko, and F. Meo, Nucl. Fusion 42, 1085, (2002).

[22] M. Brambilla, Nucl. Fusion 34, 1121, (1994).

[23] C.F. Kennel, and F. Engelmann, Phys. Fluids 9, 2377, (1966).

[24] T. Hellsten, T. Johnson, J.C. Carlson, L.-G. Eriksson, J. Hedin, M. Laxaback, and M. Mantsinen, Nucl. Fusion 44, 892, (2004).

[25] H.-U. Fahrbach (IPP Garching), private communication. 
[26] W. Suttrop, R. Budny, J.C. Cordey, G. Gowers, M. Mantsinen, et al., 28th EPS Conf. on Contr. Fusion and Plasma Physics, ECA Vol. 25A, 989, (2001).

[27] M. Brambilla, and T. Krücken, Nucl. Fusion 28, 1813 (1988).

[28] D.A. Ehst, and C.F.F. Karney, Nucl. Fusion 31, 1933, (1991).

[29] J.C. Wright, P. Bonoli, E. D’Azevedo, M. Brambilla, 15th Topical Conf. Radio Frequency Power in Plasmas, AIP Conf. Proc. 694, 511, (2003).

[30] M. Brambilla, R. Bilato, C. Maggi,H.-U. Fahrbach, W. Suttrop, and the ASDEX Upgrade Team, 16th Topical Conf. on Radio Frequency Power in Plasmas, Park City 2005 (AIP Conf. Proc. 787, S.J. Vukitch and P.T. Bonoli eds.), p. 38. 